\title{
Eficiencia del autotransporte de carga mexicano en el marco del comercio internacional: el caso del comercio de México con Canadá y Estados Unidos
}

Efficiency of the mexican trucking system within the framework of international trade: the case of the trade of Mexico with Canada and the USA

Eficiência do transporte rodoviário de carga mexicano no contexto do comércio internacional: o caso do comércio do México com o Canadá e os Estados Unidos

\author{
América Ivonne Zamora-Torres \\ Instituto de Investigaciones Económicas \\ y Empresariales de la Universidad Michoa- \\ cana de San Nicolás de Hidalgo, México. \\ E-mail: americazt@hotmail.com
}

\author{
Diana Areli Mora-Zimbron \\ Instituto de Investigaciones Económicas \\ y Empresariales de la Universidad Michoa- \\ cana de San Nicolás de Hidalgo, México. \\ E-mail:dianazimbron@hotmail.com
}

Fecha de recepción: 20/04/2017 Fecha de aceptación: 19/06/2017
Palabras clave

- Eficiencia

- autotransporte carga

- comercio internacional

\section{Resumen}

El autotransporte de carga en México, es un sector que maneja el $55 \%$ del comercio internacional mexicano y a pesar de su gran operativa comercial nacional e internacional no se cuentan con estrategias empresariales enfocadas a la generación de un sector eficiente. En la presente investigación se identificaron a partir de la revisión de literatura algunas variables que ayudarían a incrementar la eficiencia económica del autotransporte de carga mexicano con Canadá y Estados Unidos. La presente investigación descansa sobre la hipótesis de que la eficiencia del autotransporte mexicano de carga internacional, se encuentra determinada por las variables: Costos de Transporte, Calidad, Infraestructura Logística, Tecnologías de Información y Seguridad de la Cadena Logística. A fin de comprobar lo anterior se realizó un análisis de causalidad de Granger para el período 2004-2013 y un modelo de regresión (MCO) para el período 1995-2014. De acuerdo con las dos pruebas realizadas los costos y la infraestructura logística destacaron en los resultados obtenidos, como las variables más influyentes en el sector así como la existencia de un comportamiento posi- 
tivo de las operaciones comerciales por medio del autotransporte de carga mexicano con Canadá y EE.UU., sobre todo con este último, a partir de la firma del TLCAN.

\begin{abstract}
The trucking system in Mexico controls $55 \%$ of Mexican international trade and, despite its great national and international commercial operation, there are not any business strategies focused on creating an efficient sector. From the literature review, some variables which may help to increase the economic efficiency of the Mexican trucking system with Canada and the USA have been identified in this research.

This research work is based on the hypothesis which states that the efficiency of the Mexican trucking system depends on these variables: Transport Costs, Quality, Logistical Infrastructure, Information Technologies and Safety of the Logistics Chain. With the aim of confirming this, a Granger causality analysis for the period 2004-2013 and an 0LS regression analysis for the period 1994-2014 were conducted. According to the results obtained from these tests, the variables with the greatest influence on the sector are the costs and the logistical infrastructure. Another influential variable is the positive behavior of the commercial operations of the Mexican trucking system with Canada and with the USA - especially with the latter, since the signing of the NAFTA agreement.
\end{abstract}

Keywords

- efficiency

- trucking system

- international trade

\title{
Resumo
}

0 transporte rodoviário de carga no México, é um ramo que maneja $055 \%$ do comércio internacional mexicano e apesar da sua grande operativa comercial nacional e internacional não se tem estratégias empresariais focalizadas na geração de um setor eficiente. Na presente pesquisa identificaram-se a partir da revisão da literatura algumas variáveis que ajudariam a incrementar a eficiência econômica do transporte rodoviário de carga mexicano com o Canadá e os Estados Unidos. A presente pesquisa é baseada na hipótese de que a eficiência do transporte rodoviário mexicano de carga internacional, encontra-se determinada pelas variáveis: Custos de Transporte, Qualidade, Infra-estrutura Logística, Tecnologias da Informação e Segurança da Cadeia Logística. Com 0 objetivo de comprovar 0 anterior fez-se uma análise de causalidade de Granger para 0 período 2004-2013 e um modelo de regressão (MCO) para o período 1995-2014. De acordo com as duas provas realizadas, os custos e a infra-estrutura logística destacaram-se nos resultados obtidos, como as variáveis mais influentes no setor assim como a existência de um comportamento posi-

Palavras-chave

- Eficiência

- transporte rodoviário de carga

- comércio internacional tivo das operações comerciais por meio do transporte rodoviário de carga mexicano com 0 Canadá e os Estados Unidos, sobre tudo com estes últimos, a partir da assinatura do TLCAN. 


\section{Antecedentes}

En México, el mercado del autotransporte se encuentra conformado por más de 147 mil unidades económicas de las cuales 128 mil se encuentran en la modalidad Hombre-Camión y 19 mil como empresas (Ramírez, 2015).

Los «hombres-camión» se refieren a las microempresas que poseen menos de 5 vehículos y que prácticamente laboran en la informalidad (Secretaría de Comunicaciones y Transportes, 2013).

El desarrollo económico de cada país, influye de manera directa en la operación del sector transporte, en los países desarrollados las empresas transportistas afrontan regulaciones con un mayor grado de restricción, en comparación con los países menos desarrollados aunque los países con mayor desarrollo económico cuentan con un mayor número de opciones de financiamiento, permitiendo que las empresas en esos países posean una mayor facilidad de adaptación a los cambios comerciales, que en el sector transporte pueden ser: gestión y pago de las licencias, cambios en equipos de seguridad, renovación de unidades, entre otros. (Mendoza Cota y Díaz, 2003)

Las estadísticas estadounidenses del American Trucking Associations, indican que en el 2004, el $23.3 \%$ de los camiones registrados en EE.UU., se utilizaron para el comercio de carga, es decir, 31,4 millones de camiones fueron utilizados para fines comerciales (American Trucking Associations, 2017). De acuerdo con el Departamento del Transporte de EE.UU., en el 2015 el número de transportistas registrados fue de 586, 014 de los cuales el $90.8 \%$ opera 6 camiones 0 menos, a este tipo de transportistas se le denomina automotores interes- tatal, es la figura más cercana a lo que se conoce en México como hombre-camión. Las empresas grandes de autotransporte de carga estadounidense constituyen el $9.2 \%$ del total del sector (US Department of Transportation, 2017).

De acuerdo con el Programa Sectorial de Comunicaciones y Transportes la eficiencia y los costos del autotransporte de carga se ven afectados por la alta edad promedio de la flota misma que para México es de 16.5 años, mientras para EE.UU. es de 5 años (Secretaría de Comunicaciones y Transportes, 2013).

En relación con la estructura de mercado del sector transporte en México, esta es de carácter oligopólico ${ }^{1}$ como resultado de las barreras de entrada impuestas por las principales empresas en el país, dicha organización de mercado es resultado del desarrollo de estrategias empresariales por parte de las grandes empresas operadoras del servicio de transporte, designadas a restringir la operación de su competencia, inclusive a disuadir a las nuevas empresas en el sector, impidiendo la entrada de nuevos competidores (Medina Ramírez, 2011).

En relación con los combustibles utilizados en el sector, EE.UU. combina combustibles fósiles con biocombustibles en el transporte de carga, mientras México no cuenta con ese tipo de vehículos para el autotransporte de carga a nivel federal, de acuerdo con Carmona (2009), la diferencia tecnológica es una clara imposición como barrera a la libre circulación.

Según la OCDE (2007), México opera con altos costos arancelarios ${ }^{2}$ y no arancelarios $^{3}$, siendo

(1) En este caso la estructura oligopólica se refiere a la existencia de una cantidad restringida de grandes empresas en el sector.

(2) Un arancel se refiere a la Tarifa oficial para el pago de impuestos o contribuciones y tasas o derechos. Impuesto que se debe pagar por concepto de importación de bienes. Pueden ser "ad valorem" (al valor), como un porcentaje del valor de los bienes, o "específicos" como una cantidad determinada por unidad de peso o volumen. Los aranceles se emplean para obtener un ingreso gubernamental o para proteger a la industria nacional de la competencia de las importaciones. (Banco de México, 2016) Lo anterior hace referencia al costo arancelario monetario y estipulado en la comercialización de un determinado producto entre 2 países. (3) Se refieren a las normas, regulaciones y trámites burocráticos que retrasan el proceso logístico de un determinado producto de exportación o importación. Entre los costos no arancelarios se pueden encontrar, el lento procesamiento de los permisos de importación o exportación. (Rugman y Hodgetts, 1997) 
los costos de las barreras no arancelarias superiores a las arancelarias. Dicho comportamiento lo presentan los sectores de telecomunicaciones, energía y transporte. México muestra costos de barreras no arancelarias superiores a los manejados por países como Brasil, Colombia, Argentina, Japón y Canadá, entre otros.

El autor Djankov (2007) afirma que se manifiesta una disminución respecto de la eficiencia del tiempo como resultado de las barreras no arancelarias ya que por cada día adicional para transportar un producto se tiene una caída de $1 \%$ en el comercio (Djankov et al., 2007).

Debido a la cercanía con EE.UU., México maniobra más del $60 \%$ de su comercio exterior por vía terrestre, sin embargo, en gran parte de América Latina y el Caribe la tendencia es hacia el transporte marítimo. Como ejemplo se encuentra Brasil que maneja el $75 \%$ de su comercio por vía marítima (Sánchez y Ulloa, 2007).

De acuerdo con la Secretaría de Comunicaciones y Transportes en adelante SCT (2013), el autotransporte mexicano opera el $80 \%$ de la carga por medio de unidades motrices, donde las más utilizadas son: tractocamiones de tres ejes, tractocamiones de dos ejes, camiones de dos y tres ejes, entre otros. Cabe aclarar que no todas las unidades en operación se encuentran registradas ante la SCT. Para los autotransportistas de bienes mexicanos y autotransportistas privados domiciliados en México, que requieren entrar a territorio estadounidense y zonas comerciales de la frontera con EE.UU. es necesario presentar el formulario $\mathrm{OP}-1(\mathrm{MX})$, mismo que es necesario también para la obtención del registro de autorización por parte del Departamento del Transporte en EE.UU. (DOT) (Department of Transportation, 2015).

El formulario anterior es resultado del programa piloto de autotransporte transfronterizo entre México y EE.UU., cuya finalidad fue que los transportistas mexicanos obtuvieran la autorización de ingresar con sus unidades a territorio estadounidense. Dicho programa se llevó a cabo entre el 14 de octubre de 2011 y el 10 de octubre de 2014 (SCT, 2015).
El objetivo de dicho programa es brindar servicios de carga internacional de entrega de puerta-apuerta, es decir mediante la utilización del INCOTERM DDP (Entregado Con Pago de Derechos), que hasta antes de la operación de dicho programa no era utilizable debido a la falta de integración productiva entre México y EE. UU. Cualquier unidad que transporte mercancías debe llevar la carta de porte. Este documento es emitido por las empresas de transportes y los hombres-camión, y es obligatorio portarlo ya que en el mismo se indica la recepción de mercancía para ser trasladada hacia un determinado punto.

En relación con los procedimientos logísticos entre México y EE.UU., existe una marcada diferencia entre ambos países, ya que en EE.UU. el tiempo promedio de estadía de los contenedores en Recinto Fiscal es de 7 días, mientras en México es de 10 días, tomando en cuenta que el promedio de mejores prácticas mundiales es de 5 días (Instituto Mexicano para la Competitividad, 2006).

De acuerdo con el Instituto Mexicano para la Competitividad (IMCO), las empresas grandes dedicadas al autotransporte de carga participan del 13 $\%$ del mercado, las empresas medianas del $18 \%$, las empresas pequeñas del $24 \%$ y las empresas micro constituidas por la figura hombre-camión, del $45 \%$ del mercado, pudiendo observarse la importante participación del hombre-camión en el sector (Instituto Mexicano para la Competitividad, 2006).

\section{Revisión de Literatura}

El sector del autotransporte presenta evidencia literaria desde diferentes enfoques y resaltando diferentes variables, algunas de las sobresalientes son los costos. Según Sáez y Valdés (1999), afirman que los acuerdos tienen la función de reducir los costos de las relaciones económicas internacionales, ya que se encargan de proveer un conjunto de reglas aplicables a los miembros, proporcionando estabilidad y estimulando la transparencia mediante 
obligaciones específicas. Así mismo, promueven su cumplimiento mediante instrumentos vinculantes reduciendo el estímulo a desviarse de las reglas. Siendo esencial la operación en el comercio exterior de diferentes instrumentos para la reducción de futuros costos de transacción.

Coase (1988:61), define a los costos de transacción como: "los costos de medir el valor de los atributos de lo que se está intercambiando y los costos de proteger derechos y vigilar y hacer valer acuerdos".

También Solanes (1999), menciona los costos de transacción en sus investigaciones girando en torno a la logística internacional apuntando a un aumento en los costos derivado de la generación, implementación y ejecución de contratos. Desde el punto de vista comercial, los gastos logísticos constituyen un componente más importante que los aranceles dentro de los gastos comerciales totales debido a la existencia de diferentes opciones logísticas como el transporte multimodal, marítimo, terrestre, la comodalidad, entre otras. Así como a los acuerdos comerciales entre los países que ayudan a disminuir 0 eliminar los aranceles (World Bank, 2007), de acuerdo con Martínez (2008), el gasto logístico en países en desarrollo puede superar el $20 \%$ del valor del producto final.

Los costos logísticos, entre los cuales se incluyen los costos de transporte comprenden del 18 al $32 \%$ del valor del producto para los países de América Latina. Mientras que para los países integrantes de la OCDE, el costo logístico se encuentra en un promedio del $9 \%$, menor al de EE. UU. que es del $9.5 \%$ (Gonzalez, Guasch, y Serebrisky, 2007).

Gonzalez, Guasch, y Serebrisky (2007), en su artículo «Latin America: Addressing High Logistics costs and poor infraestructure for merchandise transportation and trade facilitation» realzan la importancia de los costos considerando los costos logísticos totales, los costos de transporte, los costos de transacción, los costos financieros y los costos no financieros como determinantes de la eficiencia en el transporte de mercancías.
La infraestructura es otra variable que resalta en la literatura existente del sector. Cipoletta Tomassian, Pérez Salas y Sánchez (2010), amplían la perspectiva presentada en 2007 por el Banco de México, mediante la ampliación de variables que determinan el desempeño logístico, planteando como determinantes los servicios de infraestructura de transporte para facilitar el transporte y el comercio.

Aschauer (1989) afirma que la infraestructura tiene un efecto positivo en la producción, por lo que la productividad se ve afectada de manera directa por la infraestructura en el transporte, abarcando carreteras, puertos y aeropuertos.

Por otra parte, Prud'homme (2005) compara el efecto de la infraestructura y la reducción de aranceles con el tamaño del mercado, estableciendo que ante una inversión en infraestructura se provoca una reducción de aranceles y un incremento en el tamaño del mercado en una determinada economía.

De acuerdo con la perspectiva anterior, los autores afirman que la inversión en infraestructura tiene como resultado un aumento en la especialización y en el tamaño del mercado laboral produciendo una competencia más intensa y promoviendo economías de escala.

A su vez, la calidad en la infraestructura parece ser primordial en el estudio de la eficiencia del transporte, apareciendo citada en estudios realizados por Wilmsmeier y Sanchez (2009), Arvis (2007), entre otros.

Por otro lado, varios estudios mencionan la importancia de la seguridad en el funcionamiento del autotransporte, la CEPAL (2010), divide a la seguridad relativa a la operación del transporte carretero en seguridad operacional y seguridad vial. Por seguridad operacional se refiere a las operaciones propias y complementarias del transporte encargadas de proveer un servicio seguro, incluyendo las medidas para prevención de delitos, robos y actos terroristas que afecten 0 impidan brindar el servicio (CEPAL, 2010).

Es importante mencionar que para el caso mexicano se pueden encontrar varias cifras relativas 
a seguridad operacional sin embargo, no existen cifras oficiales que representen este tipo de seguridad. La seguridad de las operaciones comerciales en el comercio exterior representa un gasto para las empresas. La Cámara Nacional del Autotransporte de Carga (CANACAR), la Asociación Mexicana de Instituciones de Seguros (AMIS), Asociación Mexicana de Seguridad Privada, Información, Rastreo e Inteligencia Aplicada (AMSIRIA), el Centro de Estudios Económicos del Sector Privado (CEESP) y la Asociación Nacional de Transporte Privado (ANTP) afirman que las empresas en México destinan del 15 al $20 \%$ a gastos inherentes a la seguridad, aunque en la mayoría de los casos, dicho monto no se manifiesta en una mejora en la seguridad (Cervantes, 2011)

Al mencionar las inversiones relativas a la seguridad se toma en cuenta la contratación de seguros de las unidades que cubran robo del vehículo, de la carga, accidentes, seguros de vida para el conductor, así como la contratación de programas satelitales para el rastreo de las unidades.

De acuerdo con Cedillo (2011), los principales factores de riesgo logístico en México son el narcotráfico (con un $30 \%$ ) y el robo (con un $25 \%$ ), de acuerdo con los resultados de su encuesta, el terrorismo y el sabotaje, fueron los factores de menor riesgo.

Las mayores violaciones al transporte dentro de la República Mexicana, se presentan en los tramos Sinaloa-Jalisco, México-Puebla, MichoacánJalisco, México-Querétaro, Querétaro-Guanajuato y Guanajuato-San Luis Potosí, así como en los estados de Nuevo León, Tamaulipas y Zacatecas, Io anterior lleva a deducir que la mayor parte del país tiene un problema de seguridad en este sector, en mayor o menor medida (Cervantes, 2012).

Ante la falta de indicadores oficiales en materia de seguridad en el sector del transporte, la SCT propuso la creación de una instancia encargada de investigar accidentes e incidentes graves de los medios de transporte de jurisdicción federal, que sucedan tanto en el autotransporte como en el transporte aéreo, ferroviario y marítimo, dicha programa se encuentra a cargo de la Agencia Federal de Investigación de Accidentes en el Transporte (AFIAT) y se enfoca en la seguridad vial (Secretaría de Comunicaciones y Transportes, 2014).

Pérez Salas (2012) sostiene que la incorporación de sistemas de transporte inteligentes (ITS, por sus siglas en inglés) como aplicación sectorial de las TICS juegan un papel primordial, ya que parece ser la conexión tecnológica que enlaza y nutre a la cadena logística, aumentando la competitividad de los participantes e incrementando la productividad de la infraestructura y servicios para la logística de cargas.

Existen autores que mencionan la importancia en la utilización de ITS, ya que éstos combinan y coordinan distintas tecnologías de control, transmisión y procesamiento de información, permitiendo mejorar la eficiencia, seguridad y sostenibilidad de los servicios de infraestructura (Pérez Salas, 2012).

Echeverría (2007) menciona la relación existente entre las TIC y la facilitación del comercio internacional definiendo a éste último como la simplificación, armonización y normalización de los procedimientos internacionales de comercio y transporte con la finalidad de crear una red comercial eficaz, así como impulsar la automatización de los procedimientos y las actividades de control asociadas a estos.

Según Dorta González (2013), la utilización de un determinado medio de transporte determina el tiempo que tendrán que estar almacenados los productos en los mercados de destino, la gestión del control de inventarios y pedidos, los embalajes a utilizar, etcétera.

Dussel Peters (2008) realiza un análisis de los procesos de exportación de varias empresas mexicanas, concluyendo que la principal limitación del transporte del comercio exterior mexicano es la incertidumbre en términos de tiempo ya que se requieren diversos permisos, existiendo incertidumbre en las aduanas en torno a la revisión exhaustiva de la mercancía, así como otros 
procesos de verificación de documentos y la mercancía, produciendo un alto nivel de incertidumbre en el tiempo de entrega del producto.

\section{Metodología}

En la presente investigación se propone la realización de un análisis de causalidad de Granger con la finalidad de conocer las relaciones causales de algunas variables que de acuerdo con estudios realizados con anterioridad aparentan tener influencia en la eficiencia económica del sector el autotransporte mexicano de carga, en el marco de los negocios internacionales.

Entre los propósitos de la econometría se encuentra el hallazgo de las relaciones causales entre variables económicas, distinguiéndolas de las relaciones espurias ${ }^{4}$ (Rodríguez Caballero, 2012).

El concepto probabilístico de causalidad se justifica en el pensamiento de que en el tiempo la causa siempre precede a los efectos. Por lo tanto, es importante conocer si una serie de tiempo causa a otra, lo que permite la predicción de valores futuros de la otra variable.

Los modelos denominados como vectores autorregresivos (VAR) de Sims (1980) y el concepto de causalidad de Granger (1969), son utilizados para la descripción de la relación causal en las series de tiempo.

Marshal (1890) planteó la idea básica del modelo causal, mediante la siguiente idea: la variable $X$ causa a $Y$ si, manteniendo todo lo demás constante, cambios en $X$ repercuten en cambios en $Y$.

El periodo de información corresponde al periodo de 2004-2013, debido a que fue el periodo donde se pudo obtener mayor información del sector, información que fue obtenida del Banco de Información Económica del INEGI, el Banco Mundial y la
Base de Datos de Transporte de América del Norte (NAICS Basis).

Gujarati y Porter (2010) afirman que aunque un análisis de regresión trate sobre la dependencia de una variable sobre otras variables, esto no es necesariamente implica causalidad. Lo anterior es la justificación para la realización de las pruebas de causalidad de Granger en esta investigación. Existen varias definiciones de "causa" mismas que tienen similitud con el concepto de "fuerza" 0 "producto" (Blalock 1961). De acuerdo con Rodríguez Caballero (2012), la definición más cercana a la definición de causalidad en el sentido de Granger es la de Feigl (1953), quien afirma que: "la causalidad es definida en términos de predictibilidad de acuerdo con la ley".

Granger (1969) afirma lo siguiente: «el tiempo no corre hacia atrás. Es decir, si un acontecimiento $\mathrm{A}$ sucede antes de un suceso $B$, es posible que $A$ cause B. Sin embargo, no es posible que B provoque $A$. En otras palabras los acontecimientos pasados pueden propiciar sucesos que ocurren en la actualidad. Lo cual no sucede con los sucesos futuros».

Para realizar la prueba econométrica de causalidad de Granger, es necesario examinar si los valores rezagados de $\boldsymbol{x}$ en la regresión de $\boldsymbol{y}$ sobre los valores rezagados de $x$ y $y$ reducen el error de varianza de forma significativa (Granger y Newbold, 1974).

Teniendo un proceso autorregresivo de orden $\boldsymbol{p}$, en $x$ y $y$ respectivamente, se utiliza la ecuación de Mínimos Cuadrados Ordinarios (MCO):

$$
\begin{aligned}
& \mathbf{x}_{\mathrm{t}}=\propto_{0}+\propto_{1} \mathbf{x}_{(\mathrm{t}-1)}+\propto_{2} \mathbf{x}_{\mathrm{t}-2}+\ldots+\propto_{\mathrm{p}} \mathbf{x}_{(\mathrm{t}-\mathrm{p})}+ \\
& \beta_{1} \mathbf{y}_{\mathrm{t}-1}+\beta_{2} \mathbf{y}_{\mathrm{t}-2}+\ldots+\beta_{\mathrm{p}} \mathbf{y}_{\mathrm{t}-\mathrm{p}}+\mathrm{u}_{\mathrm{t}}
\end{aligned}
$$

Donde la hipótesis nula establecida para esta prueba es la siguiente:

(4) Una relación espuria surge al tratar de relacionar dos variables entre las que no existe ningún tipo de relación causa-efecto mediante una regresión y se concluye erróneamente, tras la regresión, que tal regresión existe. Una de las características en la determinación de relaciones espurias reside en la obtención de un coeficiente de determinación muy elevado y un estadístico Durbin-Watson próximo a cero (Granger y Newbold, 1974). 


$$
H_{0}: \beta_{1}=\beta_{2}=\cdots=\beta_{p}=0
$$

En seguida se realiza la prueba $\boldsymbol{F}$, de acuerdo con Hamilton (1994) misma que puede estimarse también mediante MCO:

$$
S=\frac{\left(\frac{R R S S-U R S S}{P}\right)}{\frac{U R S S}{(T-2 p-1)}}
$$

Donde RRSS (por sus siglas en inglés), representa la suma de residuales al cuadrado de una autoregresión univariada no restringida mientras URSS (por sus siglas en inglés) representa la suma de residuales al cuadrado de la regresión no restringida (Rodriguez Caballero, 2012).

Por lo tanto si S es mayor que el del valor crítico, explicado en éste caso como $\boldsymbol{F}(\boldsymbol{p}, \boldsymbol{T}-2 \boldsymbol{p}-1)$ se rechaza la hipótesis nula, por lo que en ese caso no se presenta causalidad de Granger en $x$. (Rodriguez Caballero, 2012).

De acuerdo con Gujarati y Porter (2010), la prueba $\mathrm{F}$ proporciona un método general de prueba de hipótesis, en el caso de una prueba de causalidad el valor de $\mathrm{F}$ corresponde a la hipótesis que se ha establecido en un principio.

Las hipótesis establecidas en esta prueba y bajo las cuales se analizarán los resultados son las siguientes:

$$
\begin{gathered}
H_{0}: X \text { no causa a } Y \\
H_{1}: X \text { sí causa a } Y
\end{gathered}
$$

El programa econométrico utilizado realiza el cálculo para ambas variables en ambos casos, es decir, determinar si $X$ causa a $Y$ como que $Y$ cause a $X$.

De esta manera se obtiene la causalidad según Granger, C.W. (1969), misma que puede ser unidireccional 0 bidireccional. En caso de que tanto para $X$ como para $Y$ se aceptara la hipótesis alternativa $\left(\boldsymbol{H}_{1}\right)$, es decir, que $\boldsymbol{X}$ sí causa a $\boldsymbol{Y}$ de la misma manera que $Y$ sí causa a $X$, se presentaría un caso de bidireccionalidad, donde de acuerdo con Granger, C.W. (1969), ambas variables se causan.

Para determinar si se acepta la hipótesis nula 0 la hipótesis alternativa el valor crítico de F seleccionado se sitúa en el rango del .05 .

Para la presentación del siguiente modelo es necesario comprobar la estacionariedad de las variables con la finalidad de evitar la obtención de una regresión espuria. Dicha prueba se realizó para cada serie de datos mediante la prueba Dickey Fuller Aumentada (ADF por sus siglas en inglés) y se puede revisar en las Tablas 1 a 6. Posteriormente se llevó a cabo el cálculo del modelo de regresión.

Con un análisis de este tipo se obtiene la propensión marginal de las exportaciones e importaciones mexicanas, es decir, el cambio promedio de las exportaciones mexicanas enviadas a Canadá y a EE.UU. ante un cambio en el índice de precios al productos, 0 a la infraestructura ya sea carretera 0 vehicular, así como a las incidencias relacionadas con las muertes causadas por el autotransporte de carga.

\begin{tabular}{|c|c|c|c|c|c|}
\hline \multicolumn{6}{|c|}{ PRUEBAS DE RAÍZ UNITARIA - AUGMENTED DICKEYYFULLER } \\
\hline SERIES & D(LEXPAC) & $\mathrm{D}(\mathrm{LIMPDC})$ & D(LEXPAEU) & D(LIMPDEU) & $D(L V C)$ \\
\hline Test statistic & -4.917449 & -2.499871 & -3.756638 & -3.780491 & -2.910485 \\
\hline $5 \%$ level & -4.008157 & -1.974028 & -3.14492 & -1.974028 & -1.974028 \\
\hline Prob & 0.0159 & 0.0175 & 0.0183 & 0.0013 & 0.0075 \\
\hline
\end{tabular}

El nivel de significancia que se ha establecido fue del $5 \%$, por lo que cuando el valor Prob obtenido sea

Tabla 1. Prueba de raíz unitaria. Eficiencia económica 
Tabla 2. Prueba de raíz unitaria. Costos de transporte

\begin{tabular}{lr}
\hline PRUEBAS DE RAÍz UNITARIA - AUGMENTED DICKEY-FULLER \\
\hline SERIES \\
\hline Test statistic \\
\hline D\% level \\
\hline Prob
\end{tabular}

Fuente: Elaboración propia con base en el modelo econométrico (2016)
Tabla 3. Prueba de raíz unitaria. Calidad

\begin{tabular}{|c|c|c|}
\hline \multicolumn{3}{|c|}{$\begin{array}{l}\text { PRUEBAS DE RAÍZ UNITARIA - AUGMENTED DICKEY-FULLER } \\
\end{array}$} \\
\hline SERIES & $\mathrm{D}(\mathrm{LTE}, 2)$ & $\mathrm{D}(\mathrm{LIT}, 2)$ \\
\hline Test statistic & -3.776096 & -3.794847 \\
\hline $5 \%$ level & 2.043968 & -2.021193 \\
\hline Prob & 0.0042 & 0.0032 \\
\hline
\end{tabular}

Fuente: Elaboración propia con base en el modelo econométrico (2016)

Tabla 4. Prueba de raíz unitaria. Infraestructura logística

\begin{tabular}{|c|c|c|c|c|c|}
\hline \multicolumn{6}{|c|}{ PRUEBAS DE RAÍZ UNITARIA - AUGMENTED DICKEY-FULLER } \\
\hline SERIES & LUVC & $D(L P, 2)$ & D(LEAC) & LTRC & $\mathrm{D}(\mathrm{LCP})$ \\
\hline Test statistic & -4.774548 & -4.233165 & -4.363357 & -3.533203 & -3.637241 \\
\hline $5 \%$ level & -3.828975 & -1.995865 & -3.875302 & -3.11991 & -3.14492 \\
\hline Prob & 0.0119 & 0.0011 & 0.0247 & 0.0247 & 0.0223 \\
\hline
\end{tabular}

Fuente: Elaboración propia, con base en el modelo econométrico (2016)

Tabla 5. Prueba de raíz unitaria. Tecnologías de información

\begin{tabular}{lr}
\hline PRUEBAS DE RAíz UNITARIA - AUGMENTED DICKEY-FULLER \\
\hline SERIES & \\
\hline Test statistic & -3.5335 \\
\hline $5 \%$ level & -3.403313 \\
\hline Prob & 0.0427 \\
\hline
\end{tabular}

Fuente: Elaboración propia con base en el modelo econométrico (2016)

menor que 0.05 se considerará como un valor significativo confirmando la relación entre las dos o más variables en cuestión, donde la hipótesis nula es igual a cero; $\boldsymbol{H} \boldsymbol{0}: \boldsymbol{\beta}=\mathbf{0}$, mientras la hipótesis alternativa es diferente de cero; $\boldsymbol{H} \mathbf{1}: \beta \neq \boldsymbol{0}$. La naturaleza de los datos a los que se refiere esta investigación se refiere a series de tiempo con datos anuales.

Uno de los supuestos importantes para el cálculo de un modelo de regresión es que el número de
Tabla 6. Prueba de raíz unitaria. Seguridad de la cadena logística

\begin{tabular}{|c|c|c|}
\hline \multicolumn{3}{|c|}{ PRUEBAS DE RAÍz UNITARIA - AUGMENTED DICKEY-FULLER } \\
\hline SERIES & D(LA) & $\underline{D}(L M, 2)$ \\
\hline Test statistic & -3.34697 & -3.555728 \\
\hline $5 \%$ level & -3.14492 & -3.259808 \\
\hline Prob & 0.0359 & 0.0330 \\
\hline
\end{tabular}

Fuente: Elaboración propia con base en el modelo econométrico (2016)

observaciones $\boldsymbol{n}$ (datos anuales) debe ser mayor que el número de parámetros por estimar, es decir el número de variables explicativas. Por lo que se afirma el cumplimiento de dicho parámetro.

Por otra parte, es importante mencionar el Estadístico-t, mismo que se utiliza para probar la hipótesis cuyo coeficiente es igual a cero. El Estadístico-t, es diferente al valor prob, ya que este último se refiere a la probabi- 
lidad que permite decidir el rechazo 0 aceptación de la hipótesis, de que el coeficiente es igual a cero, contra la hipótesis alternativa la cual es diferente de cero.

Si el valor de Prob es menor a 0.05 se tiene evidencia para rechazar la hipótesis nula. Se puede rechazar al $5 \%$ de significancia 0 al $1 \%$ de acuerdo con el que se haya elegido. Para esta investigación se determinó un nivel de significancia del $5 \%$ para rechazar 0 aceptar la hipótesis alternativa.

\section{Resultados}

El autotransporte de carga mexicano tiene influencia de diferentes variables, mismas que fueron seleccionadas en base a la revisión de estudios empíricos. Como resultado de dicha revisión y tomando como base las hipótesis en las que se sustenta la presente investigación se determinó que la variable dependiente es la eficiencia económica del autotransporte mexicano, misma que será medida con las series de datos correspondientes al comercio con EE.UU., y Canadá así como el volumen de carga, en la tabla 7 se pueden observar las series de datos utilizadas para la eficiencia económica

Tabla 7. Series de datos que conforman la eficiencia económica

\begin{tabular}{ll}
\hline EFICIENCIA ECONÓMICA & \\
\hline Exportaciones a Canadá - Transporte carretero & EXPAC \\
\hline Importaciones de Canadá - Transporte carretero & IMPDC \\
\hline Exportaciones a Estados Unidos - Transporte carretero & EXPAEU \\
\hline Importaciones de Estados Unidos - Transporte carretero & IMPDEU \\
\hline Volumen de carga transportada en México & VC \\
\hline
\end{tabular}

Fuente: Elaboración propia (2016)

Tabla 8. Series de datos que conforman las variables independientes

\begin{tabular}{|c|c|c|}
\hline \multirow[t]{5}{*}{ Infraestructura logística } & $\underline{\mathrm{TRC}}$ & Total de la red carretera \\
\hline & $\underline{\mathrm{CP}}$ & Carreteras pavimentadas \\
\hline & UVC & Unidades vehiculares de carga \\
\hline & $\underline{E A C}$ & Empresas de autotransporte federal de carga \\
\hline & $\underline{L P}$ & Personal ocupado en el autotransporte de carga \\
\hline Seguridad de la & A & Vehículos de carga accidentados \\
\hline cadena logística & M & Muertes por accidentes de transporte de carga \\
\hline Costo de transporte & INPPAC & $\begin{array}{l}\text { Índice nacional de precios productor del autotransporte de carga } \\
\text { general }\end{array}$ \\
\hline Calidad & $\mathrm{IDL}$ & $\begin{array}{l}\text { Índice de desempeño logístico: calidad de la infraestructura } \\
\text { relacionada con el comercio y el transporte }\end{array}$ \\
\hline Tecnologías de la información & $\underline{I D C}$ & Índice de Conectividad \\
\hline
\end{tabular}


Por otra parte, las variables independientes se conformaron mediante diferentes series de datos, mismas que se presentan de manera puntual en la tabla 8.

Parar la realización de las pruebas de raíz unitaria, las series de datos se transformaron en logaritmos con ayuda y utilización del programa EViews.

Las series de datos se transforman en logaritmos naturales debido a varias razones, entre ellas, la unificación de datos ya que las unidades de medida de las series se transforman en exponentes al ser convertidas en logaritmos y por otra parte se realiza dicha transformación para simplificar los datos de las series.

Una vez que las series fueron convertidas en logaritmos se agregó la letra I al principio de las siglas que definen cada serie de datos, de esta manera se entiende que esa serie de datos ya ha sido transformada en logaritmo natural.

El valor crítico elegido para cada una de las pruebas realizadas es del $5 \%$, por lo que se aceptará la prueba siempre que el valor absoluto del $\boldsymbol{t}$-statistic sea mayor que el obtenido en el nivel del $5 \%$. Para este período las relaciones no causales se pueden observar en la tabla 9.

Tabla 9. Relaciones no causales (2004-2013)

\begin{tabular}{l}
\hline 2004-2013 \\
\hline RELACIONES N0 CAUSALES \\
\hline DLA no causa a LTRC \\
\hline DLEAC no causa a DLIMPDEU \\
\hline DLEXPAEU no causa a DLP \\
\hline DLIDC no causa a DLP \\
\hline DLM no causa a DLIMPDC \\
\hline DLVC no causa a DLEXPAEU \\
\hline DLVC no causa a DLIMPDEU \\
\hline LTRC no causa a DLVC \\
\hline LTRC no causa a LUVC \\
LUVC no causa a DLEXPAC
\end{tabular}

Fuente: Elaboración propia, con base en el modelo econométrico (2016)
En este período de datos la mayor parte de las relaciones entre variables son bidireccionales, es decir, $X$ causa a $Y$ y viceversa

Después de las pruebas de causalidad se realizó el modelo de regresión. Se puede observar una relación negativa entre las unidades vehiculares de carga y el comercio con EE.UU., Io anterior puede explicarse por el bloqueo carretero que impide que los hombres-camión tengan acceso a territorio estadounidense.

En este ámbito cabe mencionar que al comparar la infraestructura de las empresas grandes en México con las estadounidenses, las primeras son consideradas como empresas pequeñas y que los hombres- camión al no ser atendidos en frontera terminan negociando con terceros que les cobran alrededor del $30 \%$ de su utilidad (Alcántara, 2015)

Mientras que para el caso de Canadá se puede observar que la relación entre las unidades vehiculares de carga y las importaciones mexicanas provenientes de Canadá presentan una relación negativa, lo anterior debido mayormente a la distancia territorial.

En relación con la serie de datos que representa las muertes por accidentes en el autotransporte de carga, se obtuvo significancia únicamente para el caso de las Exportaciones para Canadá.

Mientras para la serie de datos que representa el total de la red carretera se obtuvo una relación positiva para las exportaciones a EE.UU., pudiendo se resultado de la circulación de las unidades mexicanas que es en gran parte por territorio mexicano, siendo pocas las unidades que cuentan con permisos para transitar por territorio estadounidense.

Por otra parte en las exportaciones a Canadá la relación es negativa con un $99 \%$ de significancia, lo anterior puede ser explicado debido a que independientemente de la infraestructura carretera mexicana, Canadá es un país que seguirá siendo abastecido por medio de unidades estadounidenses en su mayoría. 
Por otra parte el índice nacional de precios al productor presenta una relación positiva tanto para el comercio con EE.UU. como para Canadá con un $95 \%$ de significancia, a excepción de las importaciones a Canadá. El coeficiente más alto se obtuvo en la relación entre el índice nacional de precios al productor y las exportaciones mexicanas a Canadá, lo cual muestra el interés de los transportistas mexicanos para ofertar sus servicios no sólo hasta EE.UU. sino hasta Canadá. Cabe mencionar que Canadá y EE.UU. tienen una estructura empresarial muy parecida.

Tabla 10. Resultados modelo de regresión

\begin{tabular}{|c|c|}
\hline $\begin{array}{l}\text { RESULTADOS (1995-2014) } \\
\end{array}$ & \\
\hline Exportaciones a EE.UU - Transporte carretero de carga & Coeficiente \\
\hline Indice de precios productor transporte de carga (INPPAC) & 0.499378 * \\
\hline Muertes por accidentes de transporte de carga (M) & 0.064283 \\
\hline Carreteras total de la red (TRC) & 5.115515 * \\
\hline Unidades vehiculares de carga (UVC) & $-1.965609 * *$ \\
\hline Importaciones de EE.UU - Transporte carretero de carga & Coeficiente \\
\hline Indice de precios productor transporte de carga (INPPAC) & 0.731974 * \\
\hline Muertes por accidentes de transporte de carga (M) & 0.102553 \\
\hline Carreteras total de la red (TRC) & 2.798738 \\
\hline Unidades vehiculares de carga (UVC) & $-2.739797 * *$ \\
\hline Importaciones de Canadá - Transporte carretero de carga & Coeficiente \\
\hline Indice de precios productor transporte de carga (INPPAC) & 0.49877 \\
\hline Muertes por accidentes de transporte de carga (M) & 0.290863 ** \\
\hline Carreteras total de la red (TRC) & 5.662986 \\
\hline Unidades vehiculares de carga (UVC) & -1.93046 * \\
\hline Exportaciones a Canadá - Transporte carretero de carga & Coeficiente \\
\hline Indice de precios productor transporte de carga (INPPAC) & 1.59722 * \\
\hline Muertes por accidentes de transporte de carga (M) & 0.337398 ** \\
\hline Carreteras total de la red (TRC) & $-17.16312^{* *}$ \\
\hline Unidades vehiculares de carga (UVC) & 3.88637 ** \\
\hline
\end{tabular}

El Índice Nacional de Precios al Productor presenta una relación positiva tanto para el comercio con EE.UU. como para Canadá debido a que el costo por tonelada/kilómetro en México es 15 por ciento menor que en EE.UU. (Canacar, 2015), lo que parece ser positivo, sin embargo es importante destacar que la flota mexicana es mucho menor que la estadounidense y sus utilidades también son menores.
De acuerdo con la tabla 10, la variable TRC tiene los mayores coeficientes, por lo que ante un cambio en la infraestructura carretera mexicana es más evidente el cambio en el comercio con EE.UU. y Canadá.

En México, cada administración gubernamental se ha preocupado por impulsar la infraestructura mediante un crecimiento positivo. Alas unidades de carga se les permite una carga máxima de 26 
toneladas para 102 contenedores, dicha carga parece no ser revisada de manera correcta en ningún punto carretero. Ahora bien si dichas unidades pretendieran ingresar a EE.UU. con las cargas acostumbradas en México se les negaría la entrada.

Las empresas de autotransporte de carga que cuentan con oficinas en EE.UU. conocen dicha problemática y tienen un mayor control en la carga de sus unidades, sin embargo para la mayoría de los hombres-camión que no tienen acceso a territorio estadounidense el tema de la carga no es una preocupación verdadera, ya que ellos se limitan a dejar la carga en frontera, donde transfieren la responsabilidad de la mercancía y de las maniobras posteriores.

También resulta interesante el resultado obtenido de la variable INPPAC relativo al Índice de precios al productor del transporte de carga con un coeficiente del 0.49 en la primera relación, donde se entiende que a medida que el valor del índice de precios al productor en el transporte de carga se incremente en al menos 1 unidad, el incremento estimado de las exportaciones a EE.UU. por medio del autotransporte terrestre de carga incrementará en promedio en 0.49 .

Dicho incremento no llega ni a la unidad, lo cual expone los bajos costos de transporte en México, donde los ingresos para esta industria en relación con el nivel de estudio son bastante buenos aunque los operarios tienen un pesado nivel de trabajo, cuotas de tiempo que tienen que cumplirse a cabalidad, ya que el retraso de un día le genera una semana perdida al conductor. Al comparar el ingreso de un trabajador mexicano con uno estadounidense la diferencia es muy marcada, un operario mexicano percibe alrededor de $\$ 11691$ dólares estadounidenses al año en promedio, mientras que un operario estadounidense percibirá $\$ 73$ 000 dólares estadounidenses anuales y con seguro laboral garantizado, situación que en los operarios de los hombres-camión no siempre se encuentra cubierta (ITAM, 2016).
Los riesgos de trabajo como resultado del abuso de horas laboradas son accidentes viales, problemas físicos asociados a la postura que se toma al conducir, problemas físicos asociados a malos hábitos alimentarios, problemas en la salud mental, problemas de carácter psicosocial, problemas de consumo de estupefacientes, drogas y alcohol (Canacar, 2015).

En relación con la variable seguridad que en este caso se midió con la ayuda de la serie de datos de las incidencias por muertes en carretera, en el modelo de regresión no resulta representativa para el comercio internacional con EE.UU., ni se ajusta al modelo de acuerdo con el nivel de significancia y para el caso del comercio internacional con Canadá resulta significativa pero con un coeficiente pequeño, por lo que aunque resulta causal no se encuentra correlacionada. El resultado de esta variable es el mejor ejemplo de que la causalidad no implica correlación y viceversa.

\section{Conclusiones}

Se ha planteado la hipótesis que hace referencia a la eficiencia económica del autotransporte mexicano de carga, misma que se encuentra determinada por las variables: Costos de Transporte, Calidad, Infraestructura Logística, Tecnologías de Información y Seguridad de la Cadena Logística. La comprobación anterior es resultado de las pruebas de Causalidad de Granger y el modelo de regresión MCO, por lo que a continuación se describen las conclusiones obtenidas para cada una de las 5 variables propuestas.

De acuerdo con las pruebas realizadas fueron los costos y la infraestructura logística las variables que tienen verdadera influencia en la eficiencia económica del autotransporte mexicano de carga, mientras la calidad, la seguridad y la tecnología no presentaron una influencia determinante en el incremento de la eficiencia económica del autotransporte mexicano de carga, al menos mediante la realización de las pruebas econométricas realizadas. 
Lo anterior puede ser explicado debido a que como ya se mencionó el transportista mexicano centra su estrategia de venta en la oferta de un bajo costo, mismo que hasta el año pasado, se podía ofrecer con apoyo del subsidio que otorgaba el gobierno a los combustibles, subsidio que actualmente ha desaparecido provocando un incremento en el precio del combustible y por lo tanto incrementando el costo del servicio que ofrece el transportista mexicano. La eliminación del subsidio, generará que la estructura empresarial del transporte actualice sus precios debido a que el precio del combustible se actualizará diariamente a partir del mes de febrero del 2017.

Lo anterior representa una desventaja competitiva importante para el transportista mexicano ya que al parecer la única ventaja en costos que tienen estos transportistas en la actualidad es que el salario de sus operarios es 5 veces menor con respecto a un operario estadounidense (Canadian truckers, 2017).

La infraestructura medida a través del total de la red carretera, el personal ocupado y las unidades vehiculares de carga, tiene una gran influencia en el sector por obvias razones.

El total de la red carretera ha mostrado un incremento constante desde hace más de dos décadas, aunque en un porcentaje mayor se han incrementado las unidades vehiculares de carga registradas para este sector y por ende el personal ocupado.

Es importante mencionar que el aumento de las unidades vehiculares registrado en las diferentes bases estadísticas consultadas, no significa que las unidades utilizadas para este sector sean unidades nuevas, ya que a partir del 2005 se incrementó la importación de unidades usadas provenientes de
EE.UU. como resultado del huracán Katrina donde dichas unidades fueron rescatadas e introducidas al México mediante mecanismos legales amparados por el poder judicial (Barbosa , 2017).

Con el propósito de dimensionar la eficiencia económica se utilizaron las exportaciones a Canadá y a EE.UU. así como las importaciones de los mismos destinos y el volumen de carga dentro del territorio nacional mexicano. El comercio exterior con Canadá y EE.UU. mediante la utilización del transporte carretero presentó un crecimiento constante a partir de la firma del TLCAN, lo cual puede explicarse por el gran número de maquiladoras estadounidenses establecidas en el territorio mexicano, para las cuales México otorga facilidades bajo los programas IMMEX, ${ }^{5}$ PITEX, ${ }^{6}$ entre otros.

Con base a la evidencia de esta investigación, hay suficiente evidencia empírica para apoyar que las variables más influyentes en la eficiencia del autotransporte mexicano de carga son los costos y la infraestructura logística.

Es importante mencionar algunos aspectos a considerar debido al entorno económico y social, ya que aun cuando se presume que las unidades mexicanas pueden entrar a territorio estadounidense de acuerdo con el programa de libre recorrido autorizado en el 2016, también es cierto que dicha autorización, establecida partir de la firma del TLCAN no se ha respetado.

Lo anterior puede explicarse debido a presiones sindicales por parte del sector del transporte de carga estadounidense, mismos que las administraciones presidenciales han defendido sin importar lo firmado en el TLCAN.

La influencia del sindicato de camioneros estadounidenses (teamsters), ha sido importante, en

(5) El Programa IMMEX es un instrumento mediante el cual se permite importar temporalmente los bienes necesarios para ser utilizados en un proceso industrial, sin cubrir el pago del impuesto general de importación, del impuesto al valor agregado y, en su caso, de las cuotas compensatorias. (Secretaría de Economía, 2012)

(6) Programa de Importación Temporal para Producir artículos de Exportación, se refiere a las empresas registradas ante la Secretaría de Economía, a las que se les permite la importación temporal de insumos, partes, componentes, herramental, material auxiliar y maquinaria, toda vez que se comprometan a la exportación de los artículos resultantes de sus procesos productivos. (Comercio Internacional, 2017) 
el incumplimiento del TLCAN, debido a que estos últimos apoyaron de manera importante la campaña presidencial del actual mandatario estadounidense, por lo que la expectativa es que las unidades mexicanas por parte de los hombres-camión no puedan entrar a territorio estadounidense. Derivado de lo anterior no es posible tener una competencia igualitaria entre los transportistas mexicanos y los estadounidenses, debido a que finalmente el ingreso percibido por un transportista estadounidense será mayor debido a varios aspectos como el actual ajuste del precio en los combustibles en México que generará una ventaja para los transportistas estadounidenses, quienes tendrán también un menor costo en el diésel.
Dicho incremento en el precio de las gasolinas en México, provocó un incremento en el costo de los peajes, incrementando como resultado el costo del servicio del transporte de carga en México.

En el panorama actual, la diversificación de mercados es considerada una necesidad, sobre todo si se toma en cuenta la pronta renegociación que se llevará a cabo del TLCAN, así como debido al incumplimiento desde la firma de dicho tratado para el sector en cuestión. La presente investigación brindará ayuda a futuras investigaciones del sector del autotransporte de carga como soporte teórico, para poder profundizar en el conocimiento del sector, incluir otras variables de estudio e incluso otros sectores.

\section{Referencias bibliográficas}

- Alcántara, C. (05 de mayo de 2015). Hombres-camión lideran el negocio del autotransporte en México. El financiero. Recuperado de http://www.elfinanciero.com.mx/ empresas/hombres-camion-liderannegocio-del-autotransporte-enmexico.html

- American Trucking Associations (11 de febrero de 2017). Reports, trends and statistics. Recuperado en Julio 2017 de http://www.trucking. org/News_and_Information_Reports_Industry_Data.aspx

- Arvis, J. (2007). Connecting to compete: Trade Logistics in the Global Economy. The Logistics Performance Index and its indicators.

Banco Mundial. Recuperado en Julio 2017 de https://openknowledge. worldbank.org/handle/10986/24600

- Aschauer, D. (1989). Is public expenditure productive?. Journal of Monetary Economics, 23(2), 177-200.
- Barbosa, F. (2017). Propuestas para revertir el aumento de precios de los combustibles. Recuperado en Julio 2017 de Voltairenet: http://www. voltairenet.org/article195222.html

- Banco de México (2016). Glosario. Recuperado en julio 2017 de http:// www.banxico.org.mx/divulgacion/ glosario/glosario.html

- Blalock, H.M. (1961). Causal inferences in nonexperimental research. Estados Unidos: University of North Carolina Press Chapel Hill.

- Cámara Nacional del Autotransporte de Carga (Canacar). (2015). Movimiento de carga modo transporte. Recuperado en Julio 2017 de Cámara Nacional del Autotransporte de Carga: http://canacar.com.mx/stat/movimiento-carga-modo-transporte/

- Canadian truckers (1 de enero de 2017). Trucking inside Mexico. Recuperado en Julio 2017 de Truckinginfo: www.m.truckinfinfo.com
- Carmona, E. (2009). Retos y oportunidades para el transporte transfronterizo México-Estados Unidos. Norteamérica, 4(2), 173-186.

- Cedillo, G. (2011). International Logistic Summit \& Expo. Seguridad en la cadena de Suministro, Primera encuesta nacional. COMIMSA y CONACYT.

- Comisión Económica para América Latina y el Caribe (CEPAL) (2010). Seguridad en la operación del transporte de carga carretero. Boletín FAL, 285(5), 1-8. - Cervantes, M. (2012). Status of Mature Fields Rounds. Berezowsky \& Cervantes, S.C. Recuperado en Julio 2017 de https://build export.gov

- Cervantes, S. (23 de junio de 2011). México de los más inseguros para transporte de carga en AL. Directorio de Transporte, Logística y Carga T21. Recuperado en Julio 2017 de http://t21.com.mx/terrestre/2011/06/22/mexico-mas-inse- 
guros-transporte-carga

- Cipoletta Tomassian, G., Pérez Salas, G., y Sánchez, R. (2010). Políticas integradas de infraestructura, transporte y logística: experiencias internacionales y propuestas iniciales. Serie Recursos Naturales e infraestructura, 150. CEPAL. Recuperado en Julio 2017 de http://repositorio.cepal.org/handle/11362/6350

- Coase, R.H. (1988). The Firm, the Market and the Law. Estados Unidos: The University of Chicago Press.

- Comercio Internacional. (1 de Enero de 2017). Comercio Internacional. Recuperado de http://www. comerciointernacional.com.mx/ci/

- Department of Transportation. (30 de abril de 2015). Federal Motor Carrier Safety Administration. Recuperado en Julio 2017 de FMCSA: http:// www.fmcsa.dot.gov/registration/ form-op-1mx

- Djankov, S., Freund, L., y Cong, C.S. (2007). Trading on Time. Bank Policy Research, 3909. Recuperado en Julio 2017 de https://openknowledge.worldbank.org/bitstream/ handle/10986/8674/wps3909.pdf

- Dorta González, P. (2013). Transporte y Logística Internacional. Universidad de Las Palmas de Gran

Canaria. Recuperado en Julio 2017 de https://www2.ulpgc.es/hege/ almacen/download/7101/7101787/ transporte_y_logistica_internacional 2013.pdf

- Dussel Peters, E. (2008). Los costos de transporte en las exportaciones mexicanas. Internal Working Paper 13. Universidad Autónoma de México, Banco Interamericano de Desarrollo (BID) y Centro de Estudios Latinoamericanos David Rockefeller, Harvard University. Recuperado en Julio 2017 de https://publications.iadb. org/handle/11319/2514?locale-

attribute $=\mathrm{es}$

- Echeverría, M. (2007). La facilitación del comercio en las negociaciones comerciales multilaterales y bilaterales. CEPAL. Recuperado en Julio 2017 de http://www.cepal.org/es/ publicaciones/3593-la-facilitacioncomercio-negociaciones-comerciales-multilaterales-bilaterales

- Feigl, H. (1953). Notes on causality. En H. Feigl y Brodbeck (Eds.), Readings in the Philosophy of Science, (pp. 408-418). New York: Appleton Century Crofts.

- Gonzalez, J.A., Guasch, J.L., y Serebrisky, T. (2007). Latin America: Adressing High Logistics Costs and Poor Infraestructure for Merchandise Transportation and Trade Facilitation. World Bank. Recuperado en Julio 2017 de http://www.iadb.org/res/ ConsultaSanJose/files/Infrastructure_Guasch_SP_Final.pdf

- Granger, C.W. (1969). Investigating Causal Relations by Econometric Models and Cross-Spectral Methods. Econometrica, 37(3), 424-438.

- Granger, C.W. y Newbold, P. (1974). Spurious regressions in econométrics. Journal of Econometrics, 2(2), 111-120.

- Gujarati, D., y Porter, D. (2010). Econometría. México DF: McGrawHill//rwin, Inc.

- Hamilton, J.D. (1994). Time Series Analysis. Princeton, New Jersey: Princeton University Press.

- Instituto Mexicano para la Competitividad. (2006). Elementos para Mejorar la Competitividad del Transporte de Carga. México D.F.: IMCO.

- ITAM (2016). Misalario.org. Recuperado en Julio 2017 de http://www. misalario.org/main/tu-salario/com- paratusalario\#/

- Marshall, A. (1890). The Principles of Economics. History of Economic Thought Books. New York: COSIMOCLASSICS

- Martinez, R.M. (16 de junio de 2008). Hacia una Política Nacional de Logística. Taller de presentación de resultados del diseño conceptual de un esquema de sistemas de plataformas logísticas en Colombia. B0gotá, Colombia. Recuperado en Julio 2017 de http://dnplogistica.blogspot. com.ar/

- Medina Ramírez, S. (2011). Apertura fronteriza al transporte de carga mexicano: ¿Fin del problema? Comercio Exterio, 62(1), 9-11.

- Mendoza Cota, J.E., y Díaz, E. (2003). Obstáculos al comercio en el TLCAN: El caso de transporte de carga. Comercio Exterior, 53(12), 1112-1120.

- OCDE (2007). Economic Survey of Mexico. Paris: OCDE.

- Pérez Salas, G. (2012). Sistemas Inteligentes de Transporte: Oportunidades para una cadena logística sostenible y Coompetitiva. Unidad de Servicios de Infrestructura, División de Recursos Naturales e Infraestructura, CEPAL-Naciones Unidas. Recuperado en Julio 2017 de http://repositorio.cepal.org/handle/11362/7067

- Prud'homme, R. (2005). Infraestructure and development. En F. Bourguignon y B. Pleskovic (Edits.), Annual World Bank Conference on Development Economics: Lessons of Experience (pp. 153-194). Washington: Banco Mundial / Oxford University Press.

- Ramirez, D. (29 de enero de 2015). Autotransporte, actividad con el mayor costo operativo de combus- 
tible. Directorio de Transporte, Logística y Carga T21. Recuperado en Julio 2017 de http://t21.com.mx/terrestre/2015/01/29/autotransporteactividad-mayor-costo-operativocombustible.

- Rodríguez Caballero, C.V. (2012). Ensayos sobre la Granger Causalidad. Mexico: División de Ciencias Económico Administrativas, Universidad de Guanajuato.

- Rugman, A.M. y Hodgetts, R.M. (1997). Negocios internacionales: un enfoque de administración estratégica. México: McGraw-Hill

- Sáez, S., y Valdés S.J.G. (1999, abril). Chile y su política comercial "lateral". CEPAL, (67), 81-94.

- Sánchez, J. R., y Ulloa, M. (2007). Facilitación del Comercio y el Transporte en América Latina y el Caribe. ECLAC - United Nations, Boletín FAL 250. Recuperado en Julio 2017 de http:// repositorio.cepal.org/bitstream/handle/11362/37312/Bolet\%C3\%ADn\%20 FAL\%2023_es.pdf?sequence $=1$

- Secretaría de Comunicaciones y Transportes (SCT). (2013). Infraestructura de Transporte, 2013-2018. México, D.F.: SCT.

- (2014). Esquema de Cha- tarrización. México: SCT. Recuperado en Julio 2017 de Esquema de Chatarrización: gaceta.diputados.gob. $\mathrm{mx} /$ Gaceta/62/2014/feb/ConseFerr-20140218.pdf

- _ (2015). El Departamento de Transporte de los Estados Unidos anuncia su voluntad de recibir solicitudes de transportistas mexicanos para operaciones de largo recorrido. Recuperado en julio 2017 de http:// www.sct.gob.mx/despliega-noticias/ article/el-departamento-de-transporte-de-los-estados-unidosanuncia-su-voluntad-de-recibirsolicitudes-de-tr/

- Secretaría de Economía. (17 de Enero de 2012). IMMEX. Recuperado en julio 2017 de Industria Manufacturera, Maquiladora y de Servicio de Exportación: http://www.2006-2012. economia.gob.mx/comunidad-negocios/industria-y-comercio/instrumentos-de-comercio-exterior/ immex

- Sims, C.A. (1980). Macroeconomics and reality. Econometrica, 48(1), 1-48.

- Solanes, M. (1999). Servicios públicos y regulación. Consecuencias legales de fallas de mercado.
Colección Series de la CEPAL. Recuperado en Julio 2017 de http://www. cepal.org/es/publicaciones/6369servicios-publicos-regulacion-consecuencias-legales-fallas-mercado - US Department of Transportation (10 de febrero de 2017). Recuperado en Febrero 2017 de https://www. transportation.gov/

- Wilmsmeier, G., y Sanchez, R. (2009). Los desafíos del sistema de transporte en los países sin litoral de América del Sur. Serie Recursos naturales e infraestructura, 142. CEPAL. Recuperado en Julio 2017 de http://repositorio.cepal.org/handle/11362/6341

- World Bank (2007). Connecting to Compete: Trade Logistics in the Global Economy. The Logistics Performance Index and Its Indicators. Washington: International Trade Department-Transport Unit.

\section{Registro bibliográfico}

Zamora-Torres, A.I., y Mora-Zimbron, D.A. (2017). Eficiencia del autotransporte de carga mexicano en el marco del comercio internacional: el caso del comercio de México con Canadá y Estados Unidos. Revista Ciencias Económicas, 14(01), 27-43. 